\title{
Giant Pneumatocele
}

\section{ABSTRACT}

A 83-year-old woman presented to the Emergency Department with a 1-week history of dyspnea by a bulky pneumatocele. Pneumatoceles are more frequent post infectious, may be solitary or multiple and they are typically transient.

\section{CASE REPORT}

A 83-year-old woman, with heart failure, atrial fibrillation, hypertension, diabetes, chronic kidney disease and pulmonary emphysema presented to the Emergency Department with a 1 - week history of dyspnea. In the past, she was hospitalized for community acquire pneumonia. On auscultation of the chest reveals decreased breath sounds in right lung field. Laboratory was not compatible with infection. On image studies the thoracic radiography (Image 1) showed a hyper transparent circular area with well defined delimitation in the right lung field. The thoracic CT (Image 2) was performed to rule out abscess and has revealed in the posterior portion of the right lung a bulky pneumatocele measuring $13 \mathrm{~cm}$ of greater diameter and a left pleural effusion. The patient has acidemia by hypercapnic respiratory failure. The pleural effusion was transudate from heart failure. Despite she has hypercapnic respiratory failure, because the large volume pneumatocele, was decided to do not start non - invasive ventilation by the risk of increase volume and tension of pneumatocele. The patient died during hospitalization.

\section{DISCUSSION}

Pneumatocele are thin-walled parenchymal cyst, typical asymptomatic. Pneumatocelescommonly occurs after acute bacterial pneumonia, in two thirds of the cases staphylococcal pneumonia and related to delayed therapy. They also result from chest trauma and barotrauma from mechanical ventilation. Although pneumatoceles are typically asymptomatic and often disappear following resolution of the inciting event, they can cause symptoms as dyspnea and contribute to respiratory failure ${ }^{1,2}$.

\section{REFERENCES}

1 David E. Dines, MD, (1968). Diagnostic Significance of Pneumatocele of the Lung. JAMA: The Journal of the American Medical Association, 204(13), 1169.

2 Hansell DM, Bankier AA, MacMahon H, et al. Fleischner Society: glossary of terms for thoracic imaging. Radiology 2008; 246:697.

Keywords: pneumatocele, abscess, respiratory failure

\section{DIAGNOSIS \\ Giant pneumatocele}

\author{
Zarcos Palma N, da Cruz M
}

Internal Medicine Department of Centro Hospitalar Tâmega e Sousa
Image 1. Thoracic radiography showing a hyper transparent circular area with well - defined delimitation in the right lung field, corresponding to a pneumatocele.

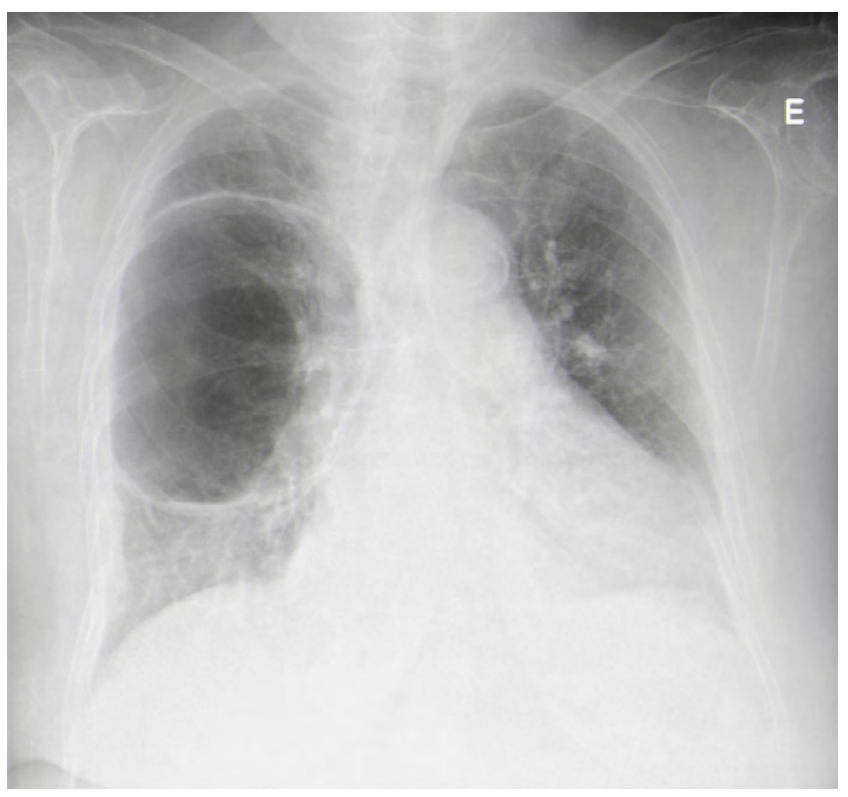

Image 2. Thoracic CT showing in the posterior portion of the right lung a bulky pneumatocele measuring $13 \mathrm{~cm}$ of greater diameter and a left pleural effusion.

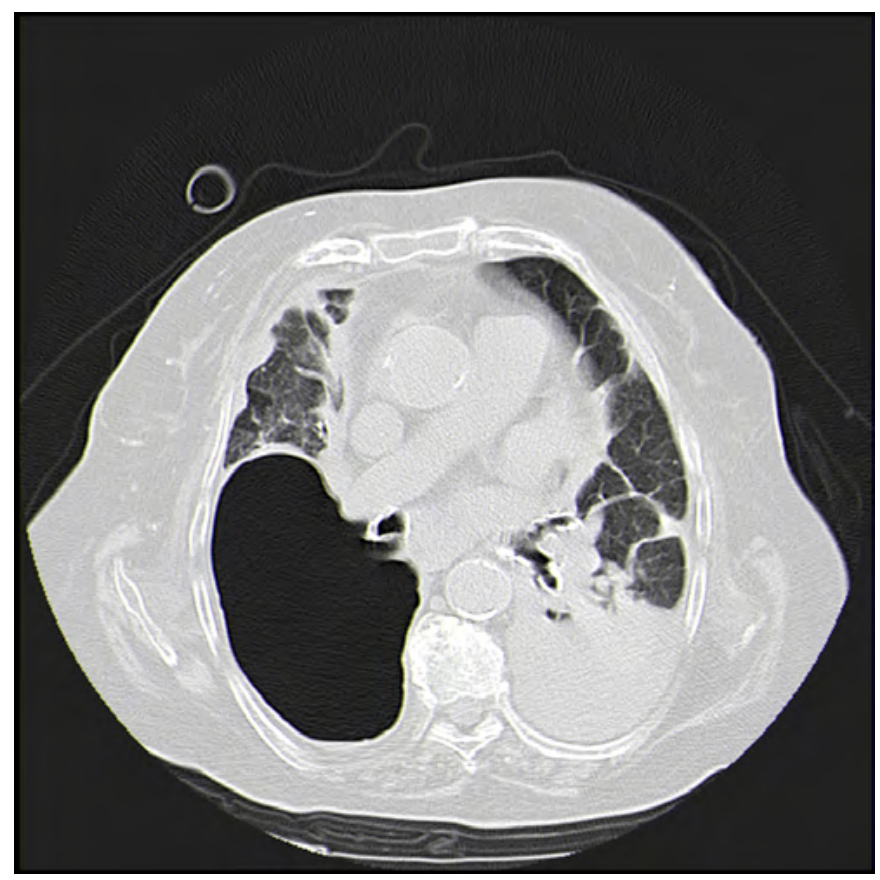

